\section{Vascular system of the giraffe}

SIR-Both the discussions of the vascular system of the giraffe you have published recently mention venous valves, but both overlook the special 'seried' valves found at the entry of major tributaries into the axillary and brachial veins, though not into the jugular. Fewer occur in the closely-related okapi and some in the jugular and femoral veins of the bactrian camel'.

These valves have a cusp proximal to the orifice of entry and another distal to it. Both are concave proximally. A number of slight differences occur in the arrangement of the cusps. Using fresh material from the camel the valve proved to be inefficient when pressure in main vein and tributary were very low, but when pressure in the main vein was raised progressively to as high as $200 \mathrm{~mm}$ of mercury regurgitation into the tributary was prevented. This pressure was higher than suggested for within the feet of the giraffe and surely for anywhere in the camel. The original workers had no access to fresh, unopened veins from a giraffe, but presumably their function in that animal would be the same and they would obviously be a great aid to the muscular pump. Why such valves should occur in the neck of the camel is hard to explain. The arterial structures in the neck of the camel $^{2}$ and both of the Giraffidae ${ }^{3}$ differ substantially.

The Old House,

R.E. REWELL

Vowchurch,

Herefordshire HR2 ORB, UK

I. Amoroso, E.C. Edhoim, O.G.\& Rewell. R.E. Proc. zool. Soc: 117,435 (1947).

2. Ask-Upmark. E. Actu psvchiat.: Suppl. 6. (1935).

3. Lawrence, W.E. \& Rewell, R.E. Proc. zool. Soc, 118. 2012 (1948)

\section{Small-particle physics and interstellar diamonds}

SIR-Lewis et al. ${ }^{1}$ have demonstrated the presence of extremely small (50 ̊) diamonds in unshocked chondrite meteorites and have convincingly argued that these diamonds were formed in a circumstellar environment as metastable condensates. No consideration has yet been given to the possibility that diamond may be the stable condensate in this size regime and that thermodynamic predictions based on the bulk properties of minerals may not be applicable to nanometre-size particulates. Unfortunately, efforts to correct for the effects of the relatively large surface/ volume ratio of particles in this regime are hampered by the lack of reliable data on the surface free energy of appropriate minerals.

Rietmeijer et al. ${ }^{2}$ have argued that in the mineral system enstatite-tridymite-fors- terite, for particle sizes $\leqslant 100 \AA$ in diameter, the mineral mixture tridymite plus forsterite is more stable than enstatite alone, despite the fact that large enstatite grains are more stable than the mixture of large tridymite plus large forsterite grains. The reversal in the relative stability of the assemblage occurs because enstatite grains have a larger surface free energy than either forsterite or tridymite grains. I propose that a similar stability reversal could occur with carbon; for example, surface effects could make diamond the stable mineral phase for very small particles. Unfortunately, this hypothesis is difficult to test as values for the surface energy of both diamond and graphite are extremely unreliable. Bulk graphite is $\sim 700$ cal

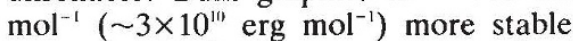
than diamond ${ }^{3}$. The molar volume ${ }^{3}$ of graphite is $5.298 \mathrm{~cm}^{3}$ whereas that of diamond is $3.417 \mathrm{~cm}^{-3}$. A reasonable estimate of the average surface free energy of a graphite sphere would be between 2,000 and 4,000 $\mathrm{erg} \mathrm{cm}^{-2}$; diamond surface energies are in the range $3,700-9,800 \mathrm{erg}$ $\mathrm{cm}^{-2}$. Given the large uncertainty in the surface free energies for diamond and graphite it might be instructive to perform an alternative calculation to see if the hypothesis of a stability reversal appears reasonable for very small carbon particles.

If we assume a value for the surface free energy of graphite and also assume that particles smaller than $50 \AA$ are more stable as diamond than as graphite, we can calculate the value of the surface free energy of diamond for which this set of assumptions would be correct. At equilibrium one mole of $50-\AA$ diameter graphite grains would coexist with one mole of 50 - $\AA$ diamond particles. But as the molar volume of graphite is larger than that of diamond this yields a surface area of $6.4 \times 10^{7} \mathrm{~cm}^{2}$ for one mole of graphite grains while the surface area of a mole of $50-\AA$ diamonds is $4.1 \times 10^{7} \mathrm{~cm}^{2}$. We have therefore

$(\text { area } \times \text { surface energy })_{\mathrm{dia}}+\Delta G_{\mathrm{dia}}=$

(area $\times$ surface energy) $)_{\text {gra }}$

As $\Delta G_{\text {dia }}$ is $\sim 3 \times 10^{10} \mathrm{erg}$, if we assume that the surface energy of graphite is $\sim 4,000$


for which equation (1) is correct is $\sim 5,500$ $\mathrm{erg} \mathrm{cm}^{-2}$. If the surface energy of graphite is only $\sim 2,000 \mathrm{erg} \mathrm{cm}^{-2}$ then the corresponding diamond surface energy is 2,400 erg $\mathrm{cm}^{-2}$. In other words, because of the larger number of less dense graphite particles needed to contain one mole of carbon, diamond's surface free energy does not actually need to be smaller than that of graphite for diamond to be more stable and it can even be significantly larger. The consequences and implications of this hypothesis will be explored in another paper ${ }^{4}$

Finally, it appears obvious that reliable measurements or calculations of the surface free energies of solid particles are necessary if we ever hope to predict correctly the behaviour of very small particle systems. It should also be obvious that conventional 'thermodynamic wisdom' does not necessarily apply to nanometrescale grains.

I wish to thank J. Hecht, G. Huss and B. Donn for several useful discussions. JosePH A. Nuth III Laboratory for Extraterrestrial Physics, NASA/Goddard Space Flight Center, Greenbelt, Maryland 20771, USA

1. Lewis, R.S., Ming, T., Wacker, J.F., Anders, E. \& Steel, E. Nature 326, 160-162 (1987).

2. Rietmeijer, F.J.M., Nuth, J.A. \& Mackinnon, I.D.R Icarus 66, 211-222 (1986).

3. Robie, R.A. \& Waldbaum, D.A. US Geol. Surv. Bull. 1259 (GPO, Washington DC, 1968)

4. Nuth, J.A. Astrophys. Space Sci. (in the press).

\section{Porosity of nuclear fuels}

SIR-In interpreting Nichols' most interesting paper ${ }^{\prime}$ on porosity in nuclear fuels, Robert Cahn ${ }^{2}$ has slipped into the trap of considering mechanisms in isolation. $\mathrm{He}$ rightly comments on the importance of grain boundary porosity formation as a contributor to fission product gas release from nuclear fuels and the micrograph he shows, produced by Hyam and his coworkers in the UK Atomic Energy Authority Laboratories at Windscale, is one of several which formed the basis of the model $^{3}$ still in use to predict such release. It is not correct, however, to deduce that fission product gas release in the columnar region is small. The high temperatures and thermal gradients that enable such grains to develop lead also to very high levels of gas release, probably by the bubble sweeping mechanism to which Nichols himself refers.

UKAEA, Windscale Laboratories,

Seascale, Cumbria CA20 IPF, UK

1. Nichols, F.A. J. nucl. Mater. 148, 92-98 (1987).

2. Cahn, R.W. Nature 328, 295-296 (1987).

3. Hargreaves. R. \& Collins, D.A. J. Br. nucl. Energy Soc. 15 311-318(1976)

\section{How many Chernobyl fatalities?}

SiR-Not to be stubborn, but for the sake of scientific truth, I would like to respond to J.H. Fremlin (Nature 327, 376; 1987). The supralinear shape of the dose-response curve both for atomic worker's cancer and for cell depression in mice is not a hypothesis but an observed fact. It correlates additional effects with additional exposures, with the obvious conclusion that the background radiation will have corresponding effects, which appears to be born out by recent careful observations. Thus Fremlin's hypothesis that background radiation somehow immunizes people against further radiation is not born out by the facts. 